Pacific Journal of Mathematic 


\title{
ON MAPPINGS FROM THE FAMILY OF WELL ORDERED SUBSETS OF A SET
}

\author{
SEYMour Ginsburg
}

A simply ordered set $E$ is called a $k$-set if there exists a simply ordered extension of the family of nonempty well ordered subsets of $E$, ordered by initial segments, into $E$. If $E$ is not a $k$-set then it is called a $k^{\prime}$-set. Kurepa $[1 ; 2]$ first discussed these sets. He showed that if $E$ is a subset of the reals and if the smallest ordinal number $\alpha$ such that $E$ does not contain a subset of order type $\alpha$ is $\omega_{1}$, then $E$ is a $k^{\prime}$-set. In particular the rationals and the reals, denoted by $R$ and $R^{+}$respectively, are both $k^{\prime}$-sets. In this paper the existence of $k$-sets and $k^{\prime}$-sets is discussed further. Theorem 7 states that each simply ordered set $E$ is a terminal segment of some $k$-set $F(E)$. It is not true, however, that each simply ordered set $E$ is similar to an initial section of some $k$-set $F(E)$ (Theorem 2). Finally, in Theorem 10 it is shown that each infinite simply ordered group is a $k^{\prime}$-set.

Following the symbolism in $[1 ; 2]$ let $E$ be a simply ordered set and $\omega E$ the family of all nonempty well ordered subsets of $E$, partially ordered as follows: For $A$ and $B$ in $\omega E, A<_{k} B$ if and only if $A$ is a proper initial segment of $B .^{1}$

Definition. A function $f$ from $\omega E$ to $E$ is called a $k$-function on $E$, if $A<_{k} B$ implies that $f(A)<f(B)$.

If there exists a $k$-function on $E$, that is, from $\omega E$ to $E$, then $E$ is called a $k$-set. If not, then $E$ is called a $k^{\prime}$-set.

TheOREM 1. If $f$ is a $k$-function on $E$, then for each nonempty well ordered subset $W$ of $E$, there exists an element $x$ in $W$ such that $f(W) \leqq x$.

Proof. Suppose that the theorem is false, that is, suppose that there exists an element $W_{1}$ in $\omega E$ with the property that $x<f\left(W_{1}\right)$ for each $x$ in $W_{1}$. Let $W_{2}=W_{1} \cup f\left(W_{1}\right)$. It is easily seen that $W_{2}$ is well ordered, $W_{1}<_{k} W_{2}, x<f\left(W_{2}\right)$ for each element $x$ in $W_{2}$, and the order type of $W_{2}$ is $\geqq 2$. Suppose that for each $0<\xi<\alpha$, $W_{\xi}$ is an element

Received October 17, 1955. Presented to the American Mathematical Society November, 1955.

$A$ is a (proper) initial segment of $B$ if $A$ is a (proper) subset of $B$ and if, for each element $z$ in $A,\{x \mid x \leqq z, x \in B\}$ is a subset of $A$. $A$ is a terminal segment of $B$ if $A$ is a subset of $B$ and if, for each element $z$ in $A,\{x \mid z \leqq x, x \in B\}$ is a subset of $\mathrm{A}$. 
of $\omega E$ such that

and

(1) $x<f\left(W_{\xi}\right)$ for each $x$ in $W_{\xi}$,

(2) $W_{\xi}<_{k} W_{v}$ for $\xi<v<\alpha$,

(3) the order type of $W_{\xi}$ is $\geqq \xi$.

Two possibilities arise.

(a) If $\alpha=\beta+1$ let $W_{\alpha}=W_{\beta} \cup f\left(W_{\beta}\right)$. By (1) and the fact that $W_{\beta}$ is well ordered, it follows that $W_{\alpha}$ is well ordered. Clearly $W_{\beta}<_{k}$ $W_{\alpha}$. Thus $f\left(W_{\beta}\right)<f\left(W_{\alpha}\right)$. It is now easy to verify that (1), (2), and (3) are satisfied for $\xi \leqq \alpha$.

(b) Suppose that $\alpha$ is a limit number. Let $W_{\alpha}=\bigcup_{\xi<\infty} W_{\xi}$. Since $W_{\xi}<_{k} W_{v}$ for $\xi<u, W_{\alpha}$ is well ordered. It is obvious that (2) and (3) are satisfied for $\xi \leqq \alpha$. Let $x$ be any element of $W_{\alpha}$. Then $x$ is in $W_{\xi}$ for some $\xi<\alpha$, thus $x<f\left(W_{\xi}\right)<f\left(W_{\alpha}\right)$. Hence (1) is also satisfied.

In this way $W_{\xi}$ becomes defined for each ordinal number $\xi$. Thus $W_{\delta}$ is defined, where $\delta$ is the smallest ordinal number such that $E$ contains no subset of order type $\delta$. This is a contradiction since $W_{\delta}$ is of order type $\geqq \delta$.

We conclude that no such set $W_{1}$ exists, that is, the theorem is true.

Suppose that $E$ is a $k^{\prime}$-set and that the ordered $\operatorname{sum}^{2} E+F$ is a $k$ set for some simply ordered set $F$. Let $f$ be a $k$-function on $E+F$. Since $E$ is a $k^{\prime}$-set, for some well ordered subset $W$ of $E, f(W)$ is not in $E$, thus is in $F$. Then $f(W) \leqq x$ for some $x$ in $W$ is false. By Theorem 1 , therefore, $f$ is not a $k$-function on $E+F$. Hence we have

THEOREM 2. If $E$ is a $k^{\prime}$-set then so is $E+F$ for every simply ordered set $F$.

The simplest example of a $k^{\prime}$-set $E$ is any infinite well ordered set. This is an immediate consequence of the following observation, whose proof is by a straightforward application of transfinite induction.

'The initial segments of an infinite well ordered set of order type $\alpha$ form a set of order type $\alpha+1$ '.

Another consequence of this observation is the following: For any infinite $k$-set $E$, the smallest ordinal number $\delta$ having the property that $E$ contains no subset of order type $\delta$, is a limit number.

Suppose that $E$ is a $k$-set and has an initial segment of $n$-elements, say $x_{0}<x_{1}<\cdots<x_{n-1}$. Letting $A_{j}=\left\{x_{i} \mid i<j\right.$ ), by a simple application of Theorem 1 , it is easily seen that $f\left(A_{j}\right)=x_{j-1}$ for each $k$-function $f$ on $E$. In other words, there is no element $x$ of $A_{j}$ such that $f\left(A_{j}\right)<x$.

2 The ordered sum $\sum_{v} E_{v}$, or $\cdots+E_{v_{1}}+\cdots+E_{v_{2}}+\cdots$, of a family of pairwise disjoint simply ordered sets is the set $E=\bigcup E_{v}$ ordered as follows: If $x$ and $y$ are in the same $E_{v}$, then $x<y$ or $y<x$ according as $x<y$ or $y<x$ in $E_{v}$. If $x$ is in $E_{v}$ and $y$ is in $E_{\nu}$ and $v<v$ in $V$, then $x<y$. 
This result cannot occur if $E$ has no first element. To be precise we have:

THEOREM 3. If $E$ is a k-set without a first element, then there exists $a k$-function $g$ such that $g(W)<x$ for each element $W$ in $\omega E$ and for some element $x$ in $W$.

Proof. Let $f$ be a $k$-function on $E$. Well order the elements of $\omega E$ into the sequence $\left\{W_{\xi}\right\}, \xi<\delta$. Suppose that $g$ is already defined for each $W_{\xi}, \xi<\theta$ (possibly other $W_{\xi}$ also) such that

(1) $g\left(W_{\lambda}\right) \leqq f\left(W_{\lambda}\right)$ for each $W_{\lambda}$ for which $g$ is defined;

(2) $g$ is not defined for $W_{\theta}$;

(3) if $g$ is defined for $W_{\gamma}$, then $g$ is also defined for each initial segment of $W_{\gamma}$;

(4) if $W_{\sigma}<_{k} W_{\tau}$ and $g$ is defined for $W_{\sigma}$ and $W_{\tau}$, then $g\left(W_{\sigma}\right)<g\left(W_{\tau}\right)$;

(5) if $g$ is defined for $W_{\xi}$, then $g\left(W_{\xi}\right)<x_{\xi}$ for some element $x_{\varepsilon}$ in $W_{\xi}$.

Let $W_{\theta}=\left\{x_{\theta, v} \mid \nu<\alpha(\theta)\right\}$ and $W_{\theta, \xi}=\left\{x_{\theta, v} \mid v<\xi\right\}$ for $0<\xi \leqq \alpha(\theta)$. Let $W_{\theta, \gamma}$ be the first $W_{\theta, \xi}$ for which $g$ is not defined: If $\gamma=1$, that is, $W_{\theta, \gamma}=\left\{x_{\theta, 0}\right\}$ let $g\left(W_{\theta, 1}\right)$ be some element of $E$ which is $<\min \left[x_{\theta, 0}, f\left(x_{\theta, 0}\right)\right]$. Such an element exists since $E$ has no first element. Suppose that $\gamma=\beta+1$, where $\beta>0$. By induction, $g\left(W_{\theta, \beta}\right)<x_{\theta, \beta}$ for some element $x_{\theta, \beta}$ in $W_{\theta, \beta}$. Let $g\left(W_{\theta, \beta+1}\right)=\min \left[x_{\theta, \beta}, f\left(W_{\theta, \beta+1}\right)\right]$. Since $W_{\theta, \beta}<W_{\theta, \beta+1}, x_{\theta, \beta}$ is not the last element in $W_{\theta, \beta+1}$. Thus $g\left(W_{\theta, \beta+1}\right)<x_{\theta, \beta+1}$ for some element $x_{\theta, \beta+1}$ in $W_{\theta, \beta+1}$. Suppose that $W_{\sigma}<_{k} W_{\theta, \beta+1}$. If $g\left(W_{\theta, \beta+1}\right)=x_{\theta, \beta}$, then $g\left(W_{\sigma}\right) \leqq$ $g\left(W_{\theta, \beta}\right)<x_{\theta, \beta}=g\left(W_{\theta, \beta}\right)$. If $g\left(W_{\theta, \beta+1}\right)=f\left(W_{\theta, \beta+1}\right)$, then

$$
g\left(W_{\sigma}\right) \leqq g\left(W_{\theta, \beta}\right) \leqq f\left(W_{\theta, \beta}\right)<f\left(W_{\theta, \beta+1}\right)=g\left(W_{\theta, \beta+1}\right) .
$$

Suppose that $\gamma$ is a limit number. Then $W_{\theta, \gamma}$ has no last element. It follows from Theorem 1 that there exists an element $x_{\theta, \gamma}$ in $W_{\theta, \gamma}$ so that $f\left(W_{\theta, \gamma}\right)<x_{\theta, \gamma}$. Let $g\left(W_{\theta, \gamma}\right)=f\left(W_{\theta, \gamma}\right)$. If $W_{\sigma}<_{k} W_{\theta, \gamma}$, then

$$
g\left(W_{\sigma}\right) \leqq f\left(W_{\sigma}\right)<f\left(W_{\theta, \gamma}\right)=g\left(W_{\theta, \gamma}\right) .
$$

By transfinite induction $g$ becomes defined for each $W_{\theta, \xi}$, thus for $W_{\theta}$ so as to satisfy (1), (3), (4), and (5). Thus $g$ becomes defined for every $W_{\xi}$. From the manner of construction, that is (4), $g$ is a $k$-function. By (5) $g$ has the property that for each element $W$ in $\omega E, g(W)<x$ for some element $x$ in $W$.

Theorem 4. If $\bar{A} \equiv \bar{B}^{3}$ and $A$ is a k-set, then so is $B$. Equivalently. if $\bar{A} \equiv \bar{B}$ and $A$ is a $k^{\prime}$-set, then so is $B$.

$3 E$ being a simply ordered set, $\bar{E}$ denotes the order type of $E . \quad \bar{A} \equiv \bar{B}$ if there exists a similarity transformation of $A$ into $B$ and a similarity transformation of $B$ into $A$. 
Proof. Let $g$ be a similarity transformation of $A$ into $B$ and $h$ a similarity transformation of $B$ into $A$. Suppose that $f$ is a $k$-function of $\omega A$ into $A$. For each well ordered subset $E$ of $B, h(E)$ is a well ordered subset of $A$ which is similar to $E$. Let $f^{*}$ be the function of $\omega B$ into $B$ which is defined by $f^{*}(E)=g f h(E)$. Clearly $g f h(C)<g f h(D)$ if $C<_{k} D$. Thus $f^{*}$ is a $k$-function, so that $B$ is a $k$-set.

Turning to the construction of $k$-sets we have

THEOREM 5. If $\left\{E_{v} \mid v \in V\right\}$ is a family of pairwise disjoint $k$-sets, and $V$ is the dual $l^{ \pm}$of a well ordered set, then the ordered sum $\Sigma E_{v}$ is a k-set.

Proof. Let $f_{v}$ be a $k$-function from $\omega E_{v}$ to $E_{v}$. Now let $A$ be a nonempty well ordered subset of $\Sigma E_{v}$. Denote by $w$ the largest element $v$ in $V$ such that $A \cap E_{v}$ is nonempty. Since $V$ is the dual of a well ordered set, $w$ exists. Let $h$ be the function which is defined by $h(A)$ $=f_{w}\left(A \cap E_{w}\right)$. There is no trouble verifying that $h$ is a $k$-function from $\omega \Sigma E_{v}$ to $\Sigma E_{v}$.

CoRollary. The dual of a well ordered set is a k-set. One particular $k$-function is the mapping which takes a well ordered subset into its largest element.

Another method of obtaining $k$-sets is to use the next result.

THEOREM 6. Let $\left\{A_{v} \mid v \in V\right\}$ be a family of pairwise disjoint simply ordered sets where $V$ is the dual of a well ordered set of order type $\alpha, \alpha$ being a limit number. Furthermore suppose that for each element $w$ in $V$, there exists a simply ordered extension $f_{w}$ of $A^{w}=\omega \sum_{v>w} A_{v}$ into $A_{w}{ }^{5}$. Then $A=\sum_{v \in V} A_{v}$ is a k-set.

Proof. Let $X$ be any nonempty well ordered subset of $A$. Let $x_{0}$ be the first element in $X . \quad x_{0}$ is in one of the sets $A_{v}$, say $A_{r}$. Since $\alpha$ is a limit number, $r$ has an immediate predecessor in $V$, say $r^{-}$. By hypothesis there exists a simply ordered extension $f_{r-}$ of $\omega A^{r-}=\omega \sum_{v>r-} A_{v}$ into $A_{r^{-}}$. Let $f(X)=f_{r^{-}}(X)$. Thus $f$ is a well defined function from $\omega A$ into $A$.

Suppose that $Y<_{k} Z$ in $\omega A$. The first element in $Y$, say $y_{0}$, is also the first element in $Z$. If $y_{0}$ is in $A_{s}$, then $f(Y)=f_{s-}(Y)<f_{s-}(Z)=f(Z)$. Thus $f$ is a $k$-function and $A$ is a $k$-set.

Now let $E_{0}$ be any simply ordered set. It is known that each

$4\left(\rho,<^{\prime}\right)$ is the dual of $(\rho,<)$ if $x<^{\prime} y$ if and only if $x>y$, for every $x$ and $y$ in $\rho$.

$5 \quad f$ is a simply ordered extension of the partially ordered set $B$ into the simply ordered set $A$ if $f$ maps $B$ into $A$ in such a manner that whenever $x<y$ in $B, f(x)<f(y)$ in $A$. 
partially ordered set has a simply ordered extension [3]. Let $f_{0}$ be a simply ordered extension of $\omega E_{0}$ into some set, say $F_{0}$. Let $E_{1}$ be a simply ordered set such that $\bar{E}_{1}=\bar{F}_{0}+\bar{E}_{0}$. Continuing by induction we obtain for each ordinal number $v$, a simply ordered extension $f_{v}$ of $\omega G_{v}$, where $\bar{G}_{v}=\cdots+\bar{E}_{\xi}+\cdots+\bar{E}_{1}+\bar{E}_{0}(\xi<\nu)$, into a simply ordered set $F_{v}$. Let $E_{v}$ be a simply ordered set such that $\overline{E_{v}}=\bar{F}_{v}+\bar{G}_{v}$. In particular, by Theorem $6, G_{\omega}$ is a $k$-set. Thus we have

THEOREM 7. Each simply ordered set $E$ is a terminal segment ${ }^{1}$ of some $k$-set $F(E)$.

REMARK. Theorem 2 shows that there exist simply ordered sets $E$ such that for no $k$-set $F(E)$ is $E$ similar to an initial segment of $F(E)$.

We now consider products of simply ordered sets, ordered by last differences.

Theorem 8. If $E$ and $F$ are k-sets, then so is $E \times F$.

Proof. Let $f$ and $g$ be $k$-functions for $E$ and $F$ respectively, and $z$ a definite element of $E$. Let $A$ be any well ordered subset of $E \times F$. Define $A_{\tau}$ to be the set $\{v \mid$ for some $u,(u, v)$ is in $A\}$. Obviously $\mathrm{A}_{\tau}$ is a well ordered subset of $F$. If $A_{\tau}$ has a last element, say $w$, let $A_{\sigma}=$ $\{u \mid(u, w)$ is in $A\}$ and let $h(A)=\left(f\left(A_{\sigma}\right), g\left(A_{\tau}\right)\right)$. If $A_{\tau}$ has no last element, let $h(A)=\left(z, g\left(A_{\tau}\right)\right)$. To see that $h$ is a $k$-function let $A<_{k} B$ in $\omega E \times F$. Since $A$ is a proper initial segment of $B$, either $A_{\tau}$ is a proper initial segment of $\mathrm{B}_{\tau}$, or else $A_{\tau}=B_{\tau}$. If the former holds, then since $g\left(A_{\tau}\right)$ $<g\left(B_{\tau}\right), h(A)<h(B)$. Suppose that the latter holds. Since $A<_{k} B$, there exists an element $(x, y)$ in $B$ which is not in $A$. Thus $A \subseteq\{(u, v)\}$ $(u, v)<(x, y),(u, v)$ in $B\}$. Since $A_{\tau}=B_{\tau}$, it follows that $y$ must be the last element of $B_{\tau}$, thus also of $A_{\tau}$. Therefore $A_{\sigma}$ and $B_{\sigma}$ exist. Since $A$ is a proper initial segment of $B, A_{\sigma}<_{k} B_{\sigma}$. As $f$ is a $k$-function, $f\left(A_{\sigma}\right)<f\left(B_{\sigma}\right)$. Hence

$$
h(A)=\left[f\left(A_{\sigma}\right), g\left(A_{\tau}\right)\right]<\left[f\left(B_{\sigma}\right), g\left(A_{\tau}\right)\right]=h(B) .
$$

REMARKs. (1) Theorem 8 is no longer true if one of the sets, either $A$ or $B$ is a $k^{\prime}$-set. This is seen by two examples.

(a) Let $E$ be a set of one element and $F$ a set order type $\omega$. Then $E \times F$ is of order type $\omega$, thus a $k^{\prime}$-set.

(b) Interchange $E$ and $F$ in (a).

(2) The conclusion of Theorem 8 may be true if one of the sets is a $k$-set and the other is not. For example

(a) Let $\bar{E}=\omega^{\omega^{*}}$ and $\bar{F}=\omega$. Then $\bar{E} \times \bar{F}=\bar{E}$, and as easily seen, $E$ 
is a $k$-set. It is also easy to show that for each ordinal number $\alpha$ and each limit number $\delta, A_{\alpha} \times B_{\delta}$ is a $k$-set, where $\bar{A}_{\alpha}=\alpha$ and $\bar{B}_{\delta}=\delta^{*}$. If $\alpha \geqq \omega$, then $B_{\delta} \times A_{\alpha}$ is a $k^{\prime}$-set.

(b) Let $A_{0}=R, f_{1}$ be a simply ordered extension of $w A_{0}$ into $B_{1}$, and $A_{-1}=\left(A_{0} \times B_{1}\right)$. In general, let $f_{n}$ be a simply ordered extension of $\omega\left(\sum_{l<n} A_{-i}\right)$ into $B_{n}$, and $A_{-n}=\left(A_{0} \times B_{n}\right)$. Let $F=\sum_{n<\omega} A_{-n}$. By Theorem 6, $F$ is a $k$-set. Then $\overline{A_{0} \times F}=\sum\left(\bar{A}_{0} \times A_{-n}\right)=\sum \bar{A}_{-n}=\bar{F}$. Thus $\mathrm{A}_{0} \times F$ is a $k$-set. It is known $[1 ; 2]$ that $A_{0}$ is a $k^{\prime}$-set.

(3) Theorem 8 is no longer true if we have a product of an infinite number of $k$-sets. For example, for each negative integer $v$ let $E_{v}=\{0$, $1\}$. Then $\Pi E_{v}$ is the set of all zero-one sequences of order type $\omega^{*}$, ordered by last differences. But $\overline{\Pi_{v} E_{v}} \equiv \lambda$, where $\lambda=\overline{R^{+}} . \quad R^{+}$is a $k^{\prime}$-set [2]. By Theorem $4, \Pi E_{v}$ is a $k^{\prime}$-set.

Question. Do there exist two $k^{\prime}$-sets $E$ and $F$ such that $E \times F$ is a $k$-set?

TheOREM 9. If $E$ is a $k^{\prime}$-set and $F$ is a simply ordered set with a first element, then $E \times F$ is a $k^{\prime}$-set.

Proof. Let $x_{0}$ be the first element of $F$ and $G=F-\left\{x_{0}\right\}$. Then $E \times F=E \times\left[\left\{x_{0}\right\}+G\right]=E \times\left\{x_{0}\right\}+E \times G$. Since $E \times\left\{x_{0}\right\}$ is a $k^{\prime}$-set, by Theorem 2 so is $E \times\left\{x_{0}\right\}+E \times G$. Hence the result.

Since $\lambda \equiv 1+\lambda$ and $\eta \equiv 1+\eta$, where $\eta=\bar{R}$, it follows from Theorem 4 and Theorem 9 that for any $k^{\prime}$-set $A, A \times R$ and $A \times R^{+}$are $k^{\prime}$-sets. In particular, Euclidean $n$-space, ordered by last differences of the coordinates of the points, is a $k^{\prime}$-set.

Theorem 10. Each infinite simply ordered group is a $k^{\prime}$-set. If $E$ is an ordered field, then there is no $k$-function from the bounded elements of $\omega E$ to $E$.

Proof. First suppose that $E$ is an ordered field. Let 1 be the multiplicative identity. For $1<x$ let $h(x)=2-1 / x$ where $2=1+1$. For $0 \leqq x \leqq 1$ let $h(x)=x$. For $x<0$ let $h(x)=-h(-x)$. Then $h$ is a similarity transformation of $E$ onto $(-2,2)$.

Suppose that $f$ is a $k$-function from the bounded elements of $\omega E$ to $E$. Let $x_{0}=z_{0}=0, z_{1}=1, x_{1}=h(1)$, and $A_{j}=\left\{x_{i} \mid i<j\right\}$ for $j=1,2$. Let $y_{1}=f\left(A_{1}\right)$ and $y_{2}=f\left(A_{2}\right)$. Clearly $y_{1}<y_{2}$. Let $z_{2}=z_{1}+\left(y_{2}-y_{1}\right)$. Thus $z_{2}$ $-z_{1}=y_{2}-y_{1}$. Let $x_{2}=h\left(z_{2}\right)$. In general suppose that for $1<\xi<\alpha, z_{\xi}$, $x_{\xi}=h\left(z_{\xi}\right), A_{\xi}=\left\{x_{v} \mid \nu<\xi\right\}$, and $y_{\xi}=f\left(A_{\xi}\right)$ are defined. Furthermore, suppose that $\left\{z_{\xi}\right\}$ and $\left\{y_{\xi}\right\}$ are strictly increasing and that $z_{\xi}-z_{1}=y_{\xi}-y_{1}$ for 
$1<\xi$. Since $E$ is a group, $z_{\xi}$ and $x_{\xi}$ are elements of $E$. Observe that $-2<x_{\xi}<2$, that is $\left\{x_{\xi}\right\}$ is a bounded sequence.

(1) Suppose that $\alpha=\beta+1$. Let $A_{\alpha}=\left\{x_{\xi} \mid \xi<\alpha\right\}, y_{\alpha}=f\left(A_{\alpha}\right), z_{\alpha}=z_{\beta}$ $+\left(y_{\alpha}-y_{\beta}\right)$, and $x_{\alpha}=h\left(z_{\alpha}\right)$. Since $A_{\beta}<_{k} A_{\alpha}, y_{\beta}<y_{\alpha}$. Thus $z_{\beta}<z_{\alpha}$ and $x_{\beta}$ $<x_{\alpha}$. Since $z_{\alpha}-z_{\beta}=y_{\alpha}-y_{\beta}$ and $z_{\beta}-z_{1}=y_{\beta}-y_{1}$, we get $z_{\alpha}-z_{1}=y_{\alpha}-y_{1}$.

(2) Suppose that $\alpha$ is a limit number. Let $A_{\alpha}=\left\{x_{\xi} \mid \xi<\alpha\right\}$ and $y_{\alpha}$ $=f\left(A_{\alpha}\right)$. Since $A_{\xi}<_{k} A_{\alpha}$, for $\xi<\alpha, y_{\xi}<y_{\alpha}$. Let $z_{\alpha}=z_{1}+\left(y_{\alpha}-y_{1}\right)$ and $x_{\alpha}=h\left(z_{\alpha}\right)$. Since $A_{\xi}<{ }_{k} A_{\alpha}$ for $\xi<\alpha, y_{\xi}<y_{\alpha}$ and thus $z_{\xi}<z_{\alpha}$ and $x_{\xi}<x_{\alpha}$. Note that $z_{\alpha}-z_{1}=y_{\alpha}-y_{1}$.

In this way, for each $\xi$ we get an $x_{\xi}$. Let $\delta$ be the smallest ordinal number such that $E$ contains no subset of order type $\delta$. The elements of the set $\left\{x_{\xi} \mid \xi<\delta\right\}$ form a strictly increasing sequence of order type $\delta$. From this contradiction we see that no such function $f$ exists.

Now suppose that $E$ is an infinite simply ordered group. Let $z_{0}=0$ and $z_{1}>0$. Let $A_{j}=\left\{z_{i} \mid i<j\right\}$ for $j=1,2$. Let $y_{1}=f\left(A_{1}\right)$ and $y_{2}=f\left(A_{2}\right)$. Repeat the procedure given above, defining $y_{\xi}$ and $z_{\xi}$ for each $\xi$, with $A_{v}=\left\{z_{\xi} \mid \xi<\nu\right\}$. We obtain a strictly increasing sequence of elements $\left\{z_{\xi}\right\}, \xi<\delta$, where $\delta$ has the same significance as above. Again we arrive at a contradiction.

REMARK. The second statement in Theorem 10 cannot be extended to hold for a group. For example, let $E$ be the group consisting of all the integers, positive, negative, and zero. The bounded, well ordered subsets of $E$ consist of the finite subsets of $E$. For this family there does exist a $k$-function, namely the function which maps each set into its maximal element.

\section{REFERENCES}

1. Kurepa, G. Sur les fonctions réeles dans la famille des ensembles bien ordonnés de nombres rationnels, Bull. Internat. Acad. Yougoslave. Cl. Sci. Math. Phys. Tech., 12 (1954), $35-42$.

2 . Fonctions croissantes dans la famille des ensembles bien ordonnés lineaires; Bulletin Scientique Yougoslavie, 2, (1954), 9.

3. Szpilrajn, E. Sur l'extension de l'ordre partiel, Fund. Math., 16, (1930), 386-389.

NORTHROP AIRCRAFT CORPORATION AND UNIVERSITY OF SOUTHERN CALIFORNIA 



\section{PACIFIC JOURNAL OF MATHEMATICS}

\section{EDITORS}

H. L. Royden

Stanford University

Stanford, California

E. Hewits

University of Washington

Seattle 5 , Washington
R. P. Dilworth

California Institute of Technology Pasadena 4, California

E. G. Straus

University of California

Los Angeles 24, California

\section{ASSOCIATE EDITORS}
E. F. BECKENBACH
C. E. BURGESS
H. BUSEMANN
H. FEDERER

\author{
M. HALL \\ P. R. HALMOS \\ V. GANAPATHY IYER \\ R. D. JAMES
}

\author{
M. S. KNEBELMAN \\ I. NIVEN \\ T. G. OSTROM \\ M. M. SCHIFFER
}

\section{J. J. STOKER \\ G. SZEKERES \\ F. WOLF \\ K. YOSIDA}

\section{SUPPORTING INSTITUTIONS}

UNIVERSITY OF BRITISH COLUMBIA CALIFORNIA INSTITUTE OF TECHNOLOGY

UNIVERSITY OF CALIFORNIA

MONTANA STATE UNIVERSITY

UNIVERSITY OF NEVADA

OREGON STATE COLLEGE

UNIVERSITY OF OREGON

UNIVERSITY OF SOUTHERN CALIFORNIA
STANFORD UNIVERSITY

UNIVERSITY OF UTAH

WASHINGTON STATE COLLEGE

UNIVERSITY OF WASHINGTON

$*$ * * *

AMERICAN MATHEMATICAL SOCIETY

CALIFORNIA RESEARCH CORPORATION HUGHES AIRCRAFT COMPANY

Mathematical papers intended for publication in the Pacific Journal of Mathematics should be typewritten (double spaced), and the author should keep a complete copy. Manuscripts may be sent to any of the editors. Manuscripts intended for the outgoing editors should be sent to their successors. All other communications to the editors should be addressed to the managing editor, E. G. Straus at the University of California, Los Angeles 24, California.

50 reprints of each article are furnished free of charge; additional copies may be obtained at cost in multiples of 50 .

The Pacific Journal of Mathematics is published quarterly, in March, June, September, and December. The price per volume (4 numbers) is $\$ 12.00$; single issues, $\$ 3.50$. Back numbers are available. Special price to individual faculty members of supporting institutions and to individual members of the American Mathematical Society: $\$ 4.00$ per volume; single issues, $\$ 1.25$.

Subscriptions, orders for back numbers, and changes of address should be sent to Pacific Journal of Mathematics, 2120 Oxford Street, Berkeley 4, California.

Printed at Kokusai Bunken Insatsusha (International Academic Printing Co., Ltd.), No. 10, 1-chome, Fujimi-cho, Chiyoda-ku, Tokyo, Japan.

\section{PUBLISHED BY PACIFIC JOURNAL OF MATHEMATICS, A NON-PROFIT CORPORATION}

The Supporting Institutions listed above contribute to the cost of publication of this Journal, but they are not owners or publishers and have no responsibility for its content or policies. 


\section{Pacific Journal of Mathematics}

\section{Vol. 6, No. 4}

1956

Seymour Ginsburg, On mappings from the family of well ordered subsets of

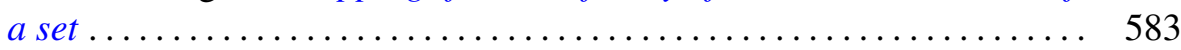

Leon Ehrenpreis, Some properties of distributions on Lie groups ......... 591

Marion K. Fort, Jr., A geometric problem of Sherman Stein ............. 607

Paul R. Garabedian, Calculation of axially symmetric cavities and jets . . . . 611

Walter Mossman Gilbert, Completely monotonic functions on cones ...... 685

William L. Hart and T. S. Motzkin, A composite Newton-Raphson gradient method for the solution of systems of equations ................. 691

C. W. Mendel and I. A. Barnett, A functional independence theorem for square matrices ................................. 709

Howard Ashley Osborn, The problem of continuous programs .......... 721

William T. Reid, Oscillation criteria for linear differential systems with complex coefficients ............................. 733

Irma Reiner, On the two-adic density of representations by quadratic

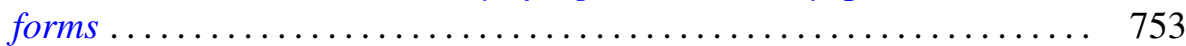

Shoichiro Sakai, A characterization of $W^{*}$-algebras .............. 763

Robert Steinberg, Note on a theorem of Hadwiger................. 775

$\mathrm{J}$. Eldon Whitesitt, Construction of the lattice of complemented ideals within

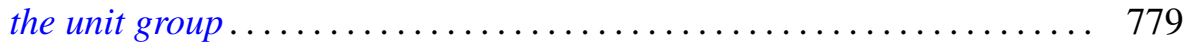

Paul Civin, Correction to "Some ergodic theorems involving two operators"... 